\title{
Propiedades físico mecánicas del Retrophyllum rospigliosii (Pilger) C.N. Page de 22 años en dos sistemas de plantación en Colombia
}

\author{
Physical mechanical properties of Retrophyllum rospigliosii (Pilger) C.N. Page \\ from 22-years-old in two plantation systems in Colombia
}

Paulo R. Portillo ${ }^{1, \star}$, Nixon Cueva ${ }^{2}$, Juan C. Sierra $^{1}$ y Ángela M. Vásquez ${ }^{1}$

Recibido: 26 diciembre 2018 | Aceptado: 27 abril 2019 | Publicado en línea: 30 junio 2019 Citación: Portillo, PR; Cueva, N; Sierra, JC; Vásquez, AM. 2019. Propiedades físico mecánicas del Retrophyllum rospigliosii (Pilger) C.N. Page de 22 años en dos sistemas de plantación en Colombia. Revista Forestal del Perú 34(1): 41-51. DOI: http://dx.doi.org/10.21704/rfp.v34i1.1284

\begin{abstract}
Resumen
Se determinaron, por primera vez en Colombia (Centro de Transferencia Tecnológica la Granja, Pensilvania - Caldas), las propiedades físico-mecánicas de la madera de pino romerón (Retrophyllum rospigliosii (Pilger) C.N. Page) de 22 años, en dos sistemas de plantación con manejo silvicultural y semilla procedente de Támesis - Antioquia. Las propiedades evaluadas fueron: contenido de humedad, densidad, contracción, flexión estática, compresión paralela y perpendicular, cizalladura radial y tangencial, dureza y extracción de clavos, de acuerdo con las Normas Técnicas Colombianas (NTC). La densidad seca al aire $\left(0.40 \mathrm{~g} / \mathrm{cm}^{3}\right)$ ubica a la madera en el grupo de las blandas o livianas; la densidad básica $\left(0.34 \mathrm{~g} / \mathrm{cm}^{3}\right)$ no permite clasificarla como estructural; su coeficiente de estabilidad dimensional la define como inestable y propensa a problemas durante el secado. Solo algunas de las propiedades presentaron diferencias significativas entre los sitios estudiados (S1-Sistema Lineal y S2-Plantación Pura), fueron inferiores a las reportadas en árboles de bosque natural con mayores dimensiones, y determinan que la madera es útil para la construcción liviana no estructural, carpintería de obra y mueblería.
\end{abstract}

Palabras clave: Conífera, propiedades físicas, propiedades mecánicas, pino romerón, plantación forestal

\footnotetext{
${ }^{1}$ Departamento de Ciencias Forestales, Universidad Nacional de Colombia - Sede Medellín, Medellín, Colombia.

${ }^{2}$ Institución de Educación Superior Colegio Integrado Nacional Oriente de Caldas (IES CINOC), Pensilvania, Colombia.

* Autor de Correspondencia: prportillob@unal.edu.co
} 


\begin{abstract}
For the first time in Colombia (Centro de Transferencia Tecnológica la Granja, Pensilvania - Caldas), the physical-mechanical properties of 22-year-old pino romerón timber trees were made (Retrophyllum rospigliosii (Pilger) C.N. Page) in two plantation systems with forestry management and seed from Támesis - Antioquia. The properties tested were: moisture content, density, contraction, static bending, parallel and perpendicular compression, radial and tangential shearing, and hardness and nail withdrawal according to Colombian Technical Standards (NTC). The air-dried density $\left(0.40 \mathrm{~g} / \mathrm{cm}^{3}\right)$ places the timber in the soft or light group; the basic density $\left(0.34 \mathrm{~g} / \mathrm{cm}^{3}\right)$ does not allow it to be classified as structural wood; and its dimensional stability coefficient defines it as unstable and prone to problems while drying. The properties showed significant differences only for some of them among the places studied (S1- Lineal Sistem and S2- Pure plantation), they were reported to being lower in natural forest trees with larger dimensions and they determined that the timber is useful for lightweight non-structural construction, carpentry work and furniture.
\end{abstract}

Key words: Conifer, physical properties, mechanical properties, pino romerón, forest plantation

\section{Introducción}

Colombia posee gran diversidad de especies promisorias para la reforestación, con alto potencial económico y de conservación, este es el caso de las Podocarpáceas, únicas coníferas nativas. Entre las especies y variedades, el pino romerón (Retrophyllum rospigliosii (Pilg.) C.N.Page) se destaca por el valor de su madera (Marín 1998), para ebanistería y construcción, pulpa para papel, tableros aglomerados, contrachapados de uso general y molduras (Veillon 1962, Arévalo y Londoño 2005, Vásquez y Alcántara 2009).

Este género posee cinco especies, entre ellas $R$. rospigliosii, en la región tropical de Suramérica (Silba 1983, Herbert et al. 2002), Venezuela, Ecuador, Perú, Bolivia, y en Colombia, en las cordilleras Central y Oriental entre 1550 y $3750 \mathrm{~m}$ de altitud (Veillon 1962, JUNAC 1981a, Torres 1988, Marín 1998). Se le conoce como pino laso, pinabete real, pinabete, pino real, pino criollo (Venezuela); ulcumano, saucecillo, romerillo blanco, romerillo macho (Perú); romerillo fino, romerillo rojo (Ecuador); pino de monte (Bolivia); pino, pino hayuelo, pino romerón, pino de pacho, pino silvestre, chaquiro crespo, chaquiro colorado (Colombia) (Veillon 1962; Aróstegui y Sato 1970; JUNAC 1981a, 1981b; Torres 1988; Zevallos 1988; Torres 1988; Acevedo y Kikata
1994; Vaca 2003; Vicuña-Miñano 2005; Zenteno-Ruíz 2007; Aranguren y Márquez 2011).

Su madera presenta albura café clara a café amarillenta (7.5YR 8/4), sin presencia de duramen o con transición gradual a duramen café claro amarillento (7.5YR 7/4); brillo moderado; olor y sabor imperceptibles; grano recto a ondulado; textura fina; veteado acentuado definido por la presencia de arcos superpuestos y bandas de coloración características; radios visibles solo con aumento de 5X en el plano transversal; anillos de crecimiento visibles solo con aumento de 5X (Vásquez 2010). Moderadamente difícil de secar al aire, con presencia de alabeos y tendencia a agrietarse y rajarse en dirección a los radios, fácil de aserrar y labrar con herramientas manuales, y así mismo de cepillar, lijar, taladrar, machihembrar, tornear y clavar, con buena retención de clavos y tornillos. Ofrece un buen desenrollado y chapas flexibles y uniformes. Es moderadamente resistente al ataque de hongos e insectos y susceptible a presentar mancha azul (Aróstegui y Sato 1970, PREDESUR 1979, JUNAC 1983).

El aprovechamiento selectivo de los bosques naturales ha llevado a que esta especie este incluida en el "Libro rojo de plantas de Colombia” (Cárdenas y Salinas 2007), por lo cual se hace necesario implementar acciones que contribuyan a la conservación y enriquecimiento de las regiones donde el pino romerón se esta- 
blecía en el pasado. En este sentido, se han realizado diferentes e importantes esfuerzos para aumentar el conocimiento sobre estos individuos en su anatomía macro y microscópica y densidad de la madera (Vásquez 2010, Vásquez et al. 2010), fenología (Gómez 2010), requerimientos de suelos y relaciones simbióticas con microorganismos como las micorrizas (Díez 2004, Díez et al. 2008), sistemas de plantación a modo de conservación ex situ, bancos de germoplasma, ensayos de procedencias y biología reproductiva (Cueva y Trujillo 2016), distribución, ecología, vivero y silvicultura (Marín 1998, Cueva et al. 2013).

El conocimiento de las propiedades de una madera ligado al manejo silvicultural de la especie son aspectos que contribuyen decisivamente a determinar su potencial, mejorar su aprovechamiento y obtener una mejor comprensión de la magnitud de la influencia de estas propiedades en su procesamiento (de Lima et al. 2016, Vieira et al. 2016) y utilización (Lima et al. 2014), necesidades apremiantes en el caso de nuestras especies nativas, cuyo uso adecuado se ve limitado por la falta de conocimiento científico sobre sus propiedades físico-mecánicas y la obtención de valores que permitan caracterizarlas y expandir sus posibilidades de utilización en el sector forestal nacional (Pedroso et al. 2016). En Perú y Ecuador, estas propiedades de la madera de pino romerón fueron estudiadas en árboles de bosque natural sin edad conocida (JUNAC 1981b, Aróstegui y Sato 1970), y en estado verde $(\mathrm{CH}>30$ $\%)$ en una plantación de 32 años bajo sistema agroforestal con café por García et al. (2017). En Colombia no se tienen registros de investigaciones realizadas para establecer las propiedades de la madera de árboles plantados, razón por la cual el presente estudio reviste especial importancia pues su objetivo principal fue determinar, por primera vez, las propiedades físico-mecánicas de la madera de árboles de pino romerón de 22 años en dos sistemas de plantación con manejo silvicultural, S1 (Sistema Lineal) y S2 (Plantación Pura), como la base para aumentar su conocimiento y mejorar su utilización.

\section{Materiales y Métodos}

\section{Selección de sitios de estudio}

En el Centro de Transferencia Tecnológica la Granja (Pensilvania - Caldas), entre cinco ensayos existentes de pino romerón, todos con edad de 22 años y procedentes de semilla de árboles de Támesis - Antioquia, fueron seleccionados dos sitios, por la presencia de individuos con mejor forma y mayores dimensiones.

Sitio 1 (S1): sistema lineal ( $5^{\circ} 22^{\prime} 47.67^{\prime \prime} \mathrm{N}$ $\left.75^{\circ} 08^{\prime} 15.02^{\prime \prime} \mathrm{O}\right)$ establecido en 1.73 ha como barrera viva forestal y árboles de altura total entre 14.75 y $17.75 \mathrm{~m}$ y DAP entre 28.2 y $34.4 \mathrm{~cm}$ (Cuadro 1). A $1937 \mathrm{~m}$ de altitud, suelos franco arenosos de $35 \mathrm{~cm}$ de profundidad, relieve ondulado con pendiente de $45 \%$, distancia promedia de siembra de $2.5 \mathrm{~m}$ y callejones de 17 $\mathrm{m}$, con dos podas de formación y un aclareo.

Sitio 2 (S2): plantación pura ( $5^{\circ} 22^{\prime} 45.55^{\prime \prime}$ $\mathrm{N}-75^{\circ} 08^{\prime} 20.97^{\prime \prime} \mathrm{O}$ ) establecida en 2.27 ha $\mathrm{y}$ árboles de altura total entre 15.25 y $18.25 \mathrm{~m} \mathrm{y}$ DAP entre 26 y $33 \mathrm{~cm}$ (Cuadro 1). A $1962 \mathrm{~m}$ de altitud, suelos franco arenosos de $50 \mathrm{~cm}$ de profundidad, relieve escarpado con pendiente fuerte de $85 \%$ y distancia de siembra promedia de $4.6 \mathrm{~m}$, con tres podas de formación y dos aclareos, el primero en 2005 a la edad de 11 años e intensidad del $20 \%$ y el segundo en 2008 a la edad de 14 años, con intensidad del $10 \%$ (Cueva et al. 2013).

\section{Selección de árboles de pino romerón}

En ambos sitios se realizó una preselección de árboles con DAP y altura mínima de $26 \mathrm{~cm}$ y $14.5 \mathrm{~m}$, respectivamente, y entre los mismos, se eligieron ocho en cada sitio, por ser los individuos con mejor forma (copa angosta, ramas delgadas, fuste recto y cilíndrico) y mejores condiciones sanitarias de acuerdo con la NTC 787 (Icontec 1974a). Estos fueron apeados a 10 $\mathrm{cm}$ del suelo y luego de su desrame, de cada uno se obtuvieron tres trozas de $3 \mathrm{~m}$, para un total de cuarenta y ocho, que fueron marcadas con un código alfanumérico de la siguiente manera: S1 y S2 para identificar los sitios seleccionados, seguidos de un número de uno a ocho para registrar el árbol y posteriormente, 
de las letras A, B y C, para diferenciar las trozas de base a copa. La madera se transportó a las instalaciones del Laboratorio de Productos Forestales Héctor Anaya López de la Universidad Nacional de Colombia Sede Medellín.

\section{Elaboración de probetas y determinación de propiedades físicas y mecánicas}

Las trozas fueron seccionadas en tablones de $6 \mathrm{~cm}$ de espesor, la mayoría de los cuales, fueron llevados a un horno de secado a $60^{\circ} \mathrm{C}$ por diez días para agilizar la pérdida de humedad en la madera, y seguidamente almacenados al aire libre hasta un contenido de humedad entre $18 \mathrm{y}$ $20 \%$. Algunos tablones en estado verde fueron utilizados para extraer las probetas necesarias para la determinación de las propiedades físicas de la madera. Posteriormente, se cortaron listones, y de estos las probetas que luego se seleccionaron entre aquellas libres de defectos y con buena orientación conforme a la NTC 301 (Icontec 1969) y NTC 3377 (Icontec 2004a).

La determinación de las propiedades físicas se realizó en dos probetas por troza, seis probetas por árbol y ensayo y 94 totales (46 para S1 y 48 para S2), a través de los ensayos de contracción (radial, tangencial y volumétrica) y la relación contracción tangencial - radial (T/R) o coeficiente de estabilidad dimensional (CED) NTC 701 (Icontec 2003a); densidad (verde, seca al aire, anhidra, básica) NTC 290 (Icontec 1974b); y contenido de humedad (verde y seco al aire) NTC 206-1 (Icontec 2006).

La determinación de las propiedades mecánicas se realizó en una máquina Tinius Olsen con capacidad de $15000 \mathrm{~kg}$, accesorios para cada ensayo y la madera en condición seca al aire (contenido de humedad cercano al 12 $\%)$, en dos probetas por troza, cuatro por árbol y ensayo, para un total de 431 (220 para S1 y 211 para S2), extraídas principalmente de las trozas A y B y solo en algunos casos de la C, debido a la presencia de numerosos nudos como el defecto más relevante.

Se realizaron ensayos de flexión estática NTC 663 (Icontec 2003b) para evaluar el comportamiento de la madera al ser sometida a un es-

\begin{tabular}{cccc}
\hline Sitio & Árbol & Ht (m) & DAP (cm) \\
\hline \multirow{4}{*}{ S1 } & 1 & 17.00 & 29.0 \\
& 2 & 17.00 & 34.4 \\
& 3 & 17.50 & 29.6 \\
& 4 & 17.75 & 30.0 \\
& 5 & 15.50 & 32.1 \\
& 6 & 17.00 & 33.2 \\
& 7 & 14.75 & 28.2 \\
S2 & 8 & 16.75 & 33.1 \\
\hline & 1 & 17.00 & 26.0 \\
& 2 & 17.00 & 27.9 \\
& 3 & 15.25 & 25.6 \\
& 4 & 18.00 & 28.9 \\
& 5 & 18.25 & 29.6 \\
& 6 & 17.75 & 30.1 \\
& 7 & 17.00 & 26.9 \\
& 8 & 18.00 & 33.0 \\
\hline
\end{tabular}

Cuadro 1. Dimensiones de los árboles seleccionados, altura total (Ht) y diámetro a la altura del pecho (DAP), en S1 y S2.

fuerzo que trata de causar una curvatura en su longitud; de compresión, al someter la madera a dos fuerzas en la misma dirección que producen un aplastamiento de su estructura en sentido paralelo NTC 784 (Icontec 2004b) y perpendicular al grano NTC 785 (Icontec 2004c); de cizalladura radial y tangencial NTC 775 (Icontec 2004d) para establecer la resistencia interna de las células al someterlas a un esfuerzo aplicado sobre una porción de la pieza; de dureza NTC 918 (Icontec 2004e) para evaluar la resistencia de la madera a la penetración de otro objeto; y de extracción de clavos NTC 951 (Icontec 2004f) para medir la fortaleza de las uniones y su tendencia a la formación de grietas. Resultados que fueron ajustados al $12 \%$ de contenido de humedad, excepto en el ensayo de extracción de clavos.

\section{Análisis estadístico}

Se calcularon estadísticos descriptivos básicos (media, intervalo de confianza, desviación estándar y coeficiente de variación) y se realizó 
un análisis de varianza de un factor para la diferencia de medias entre los sitios, mediante el programa STATGRAPHICS (StatPoint Technologies 2009).

\section{Resultados}

\section{Propiedades físicas}

El Cuadro 2 muestra los valores medios obtenidos en la determinación para las propiedades físicas de la madera de pino romerón de plantación, en S1 (Sistema Lineal) y S2 (Plantación Pura).

Las densidades verde, seca al aire, seca al horno y básica promedias fueron $0.76,0.40$, 0.37 y $0.34 \mathrm{~g} / \mathrm{cm} 3$, respectivamente (Cuadro 2). Sin diferencias significativas entre sitios ( $P$ $>0.05$ ), excepto para la densidad verde. Tampoco se encontraron diferencias significativas entre los valores obtenidos para las contracciones normales y totales ni para el coeficiente de estabilidad dimensional (CED), entre sitios $(\mathrm{P}>0.05)$.

\section{Propiedades mecánicas}

El Cuadro 3 muestra los valores medios obtenidos en la determinación de las propiedades mecánicas (ajustados al $12 \%$ ), de la madera de pino romerón de plantación, en S1 (Sistema Lineal) y S2 (Plantación Pura).

En flexión estática los valores promedio obtenidos para la población en el Esfuerzo al Límite Proporcional (ELP), Módulo de Ruptura (MOR) y Módulo de Elasticidad (MOE) fueron $337.02,499.01$ y $55320 \mathrm{~kg} / \mathrm{cm}^{2}$, respectivamente (Cuadro 3). Con diferencias significativas $(\mathrm{P}<0.05)$ entre sitios para el ELP y MOR, $y$ sin diferencias para el MOE.

Para la compresión paralela los valores de ELP, MOR y MOE promedios para la población fueron $162.92,227.92$ y $75370 \mathrm{~kg} / \mathrm{cm}^{2}$, respectivamente. Sin diferencias significativas entre sitios $(\mathrm{P}>0.05)$ para el ELP y MOR, y con diferencias para el MOE. En sentido perpendicular el ELP promedio obtenido fue de 52.24 $\mathrm{kg} / \mathrm{cm}^{2}$, sin diferencia significativa entre sitios $(\mathrm{P}>0.05)$ (Cuadro 3).
El valor promedio para el MOR en las cizalladuras radial y tangencial fue 49.29 y $56.38 \mathrm{~kg} /$ $\mathrm{cm}^{2}$, respectivamente. Sin diferencias significativas entre sitios $(P>0.05)$ en dirección radial $y$ con diferencias para la tangencial $(\mathrm{P}<0.05)$ (Cuadro 3).

En dureza el valor promedio para la población resultó superior en los extremos $(337.90 \mathrm{~kg})$ que en los lados $(212.03 \mathrm{~kg})$, sin diferencia significativa para los mismos entre sitios $(\mathrm{P}>0.05)$ (Cuadro 3).

En el ensayo de extracción de clavos la resistencia poblacional media obtenida en los planos longitudinales radial y tangencial y en los extremos fue de $43.85,53.25$ y $41.83 \mathrm{~kg}$, respectivamente, sin presencia de grietas, y con diferencia significativa para los mismos entre sitios $(\mathrm{P}<0.05)($ Cuadro 3$)$.

\section{Discusión}

\section{Propiedades físicas}

El valor promedio para la densidad seca al aire en la madera de pino romerón $\left(0.40 \mathrm{~g} / \mathrm{cm}^{3}\right)$ (Cuadro 2), se encuentra en el límite inferior del rango utilizado por JUNAC (1984) para las blandas o livianas, con densidades secas al aire entre 0.40 y $0.72 \mathrm{~g} / \mathrm{cm}^{3}$, de utilidad general para carpintería, construcción no estructural, mueblería y decorativas. Poco durables en climas tropicales, pero pueden usarse con éxito si son tratadas convenientemente.

La densidad básica media para la población de $0.34 \mathrm{~g} / \mathrm{cm} 3$ (Cuadro 2), no permite clasificar a la madera de pino romerón en los grupos estructurales A, B o C estipulados por JUNAC (1984), es similar a la encontrada por García et al. (2017) $\left(0.35 \mathrm{~g} / \mathrm{cm}^{3}\right)$ en árboles procedentes de una plantación de 32 años en Perú y clasificada como baja (entre 0.30 y $0.40 \mathrm{~g} / \mathrm{cm}^{3}$ ) por Aróstegui (1982). Es inferior a los valores obtenidos en individuos de bosque natural con edad desconocida por JUNAC (1981b) en Ecuador $\left(0.57 \mathrm{~g} / \mathrm{cm}^{3}\right)$, por Aróstegui y Sato (1970) en Perú $\left(0.41 \mathrm{~g} / \mathrm{cm}^{3}\right)$ y por Vásquez (2010) en Colombia $\left(0.59 \mathrm{~g} / \mathrm{cm}^{3}\right)$; y más cercana al valor hallado en especímenes de menor edad y dimensiones que en el presente estudio 
Propiedades físico mecánicas del Retrophyllum rospigliosii (Pilger) C.N. Page de 22 años en dos sistemas de plantación en Colombia

\begin{tabular}{|c|c|c|c|c|c|}
\hline & Ensayo & S1 & $\mathbf{S 2}$ & $\begin{array}{c}\text { Media } \\
\text { población }\end{array}$ & CV\% \\
\hline Contenido de & Verde $(>30 \%)$ & 133 & 119 & 126 & 18,18 \\
\hline \multirow[t]{2}{*}{ humedad (\%) } & Seco al aire (10-14\%) & 12 & 11 & 12 & 2,67 \\
\hline & Verde & 0.79 & 0.73 & 0.76 & 11,30 \\
\hline Densidad (g/ & Seca al aire & 0.41 & 0.39 & 0.40 & 9,44 \\
\hline \multirow[t]{2}{*}{$\left.\mathbf{c m}^{3}\right)$} & Seca al horno & 0.38 & 0.37 & 0.37 & 9,86 \\
\hline & Básica & 0.34 & 0.33 & 0.34 & 9,15 \\
\hline Contracción & Radial & 1.97 & 2.11 & 2.04 & 29,80 \\
\hline $\begin{array}{c}\text { normal (\%) } \\
\text { (verde a seco al }\end{array}$ & Tangencial & 3.97 & 4.23 & 4.10 & 27,26 \\
\hline aire) & Volumétrica & 7.83 & 8.02 & 7.93 & 21,37 \\
\hline Contracción & Radial & 3.13 & 3.28 & 3.20 & 27,31 \\
\hline total $(\%)$ & Tangencial & 5.56 & 5.97 & 5.76 & 21,47 \\
\hline \multirow{2}{*}{$\begin{array}{c}\text { (verde a seco al } \\
\text { horno) }\end{array}$} & Volumétrica & 10.82 & 11.33 & 11.08 & 16,89 \\
\hline & Relación T/R (CED) & 1.85 & 1.91 & 1.88 & 26,33 \\
\hline
\end{tabular}

CED: coeficiente de estabilidad dimensional

Cuadro 2. Valores medios para las propiedades físicas de la madera de pino romerón de plantación, en S1 y S2.

(ocho años, $7.5 \mathrm{~m}$ de altura y $20.8 \mathrm{~cm}$ de DAP), provenientes de bosque plantado en Colombia $\left(0.37 \mathrm{~g} / \mathrm{cm}^{3}\right)$ y estudiados por Vásquez y Alcántara (2009).

De acuerdo a PROEXPO (1970), su contracción volumétrica normal es alta $(7.93 \%)$ y la total es moderada (11.08\%) y mayor a la encontrada por García et al. (2017) (9.01\%). Las contracciones radial y tangencial totales $(3.20 \mathrm{y}$ $5.76 \%)$ concuerdan con las obtenidas por JUNAC (1981b) (3.2 y 5.7\%) y por García et al. (2017) (2.91 y 5.98\%), pero resultan inferiores a las encontradas por Aróstegui y Sato (1970) (3.6 y $7.3 \%)$.

El coeficiente de estabilidad dimensional (CED) para la población (1.88), es equivalente a los obtenidos por JUNAC (1981b) (1.9) y Aróstegui y Sato (1970) (2.0) y permite clasificar la madera de pino romerón como desfavorable (PROEXPO 1970); propensa a presentar problemas durante el proceso de secado (JUNAC 1989) y alto índice de distorsiones y alabeos (Etchenique et al. 1975); aunque Ordóñez et al. (1990) la clasifican como una madera estable $(1,5-2,0)$.

\section{Propiedades mecánicas}

En el ensayo de flexión estática los valores medios para la población de ELP, MOR y MOE $\left(337.02,499.01\right.$ y $\left.55320 \mathrm{~kg} / \mathrm{cm}^{2}\right)$, fueron inferiores a los reportados por Aróstegui y Sato (1970) $\left(442,788\right.$ y $\left.109000 \mathrm{~kg} / \mathrm{cm}^{2}\right)$ y por JUNAC (1981b) (423, 1016 y $\left.96000 \mathrm{~kg} / \mathrm{cm}^{2}\right)$ 


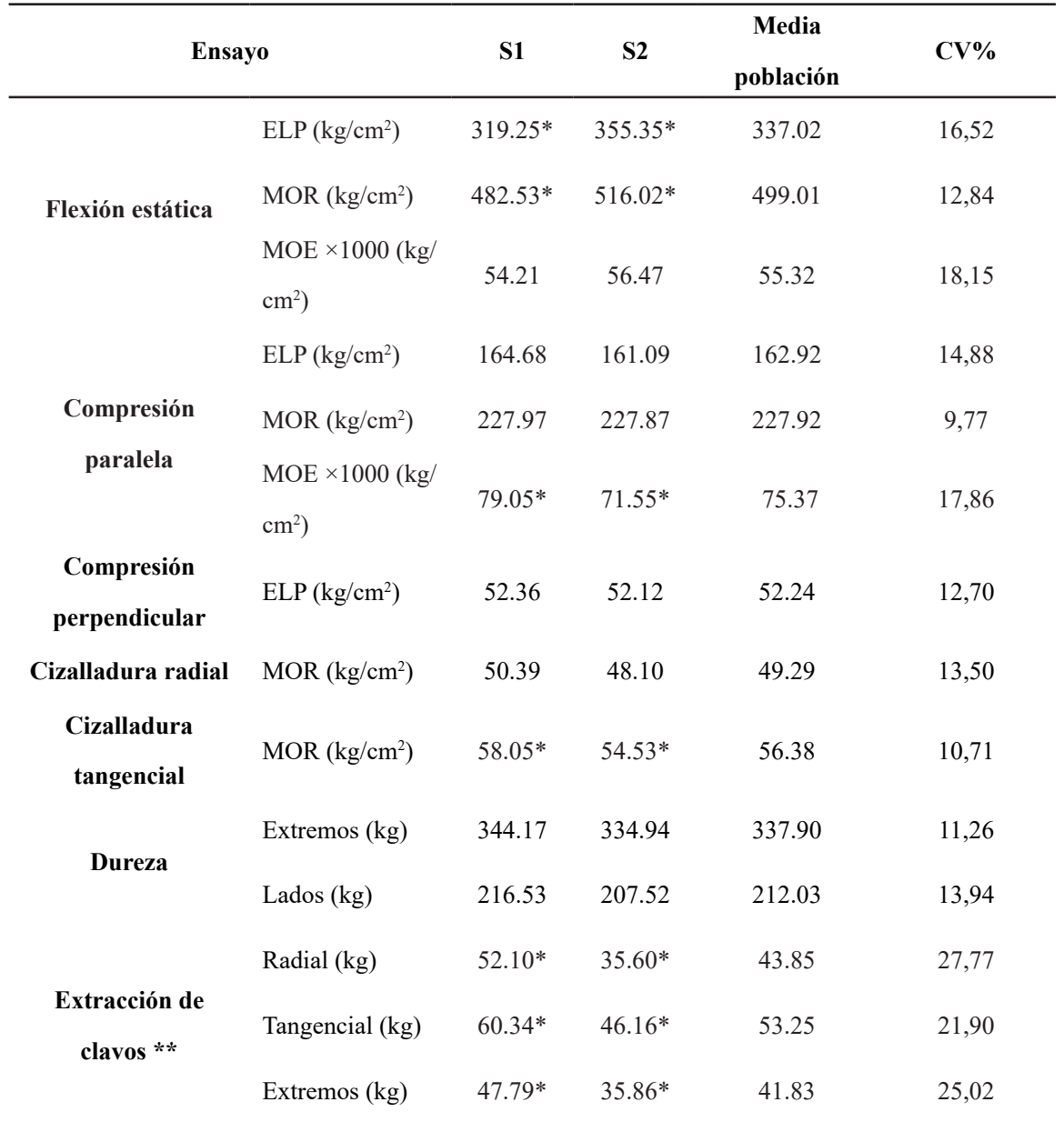

* Diferencia significativa $(\mathrm{P}<0.05), * *$ Valores en madera seca al aire (contenido de humedad cercano al $12 \%)$

Cuadro 3. Valores medios ajustados al $12 \%$ para las propiedades mecánicas de la madera de pino romerón de plantación, en S1 y S2.

(Cuadro 3), en árboles de bosque natural sin edad conocida y mayores dimensiones que en el presente estudio.

En compresión paralela el ELP y el MOE promedios para la población $\left(162.92 \mathrm{~kg} / \mathrm{cm}^{2} \mathrm{y}\right.$ $75370 \mathrm{~kg} / \mathrm{cm}^{2}$ ) fueron inferiores a los obtenidos por Aróstegui y Sato (1970) $\left(246 \mathrm{~kg} / \mathrm{cm}^{2}\right.$ y $\left.109700 \mathrm{~kg} / \mathrm{cm}^{2}\right)$, al igual que el MOR $(227.92$ $\mathrm{kg} / \mathrm{cm}^{2}$ ) al ser comparado con los reportados por este autor $\left(412 \mathrm{~kg} / \mathrm{cm}^{2}\right)$ y por JUNAC (1981b) $\left(473 \mathrm{~kg} / \mathrm{cm}^{2}\right)$ (Cuadro 3).
Los valores obtenidos para el MOE en ambos ensayos concuerdan con lo expresado en JUNAC (1984), con respecto a que en maderas tropicales el MOE en compresión paralela es superior al MOE en flexión estática.

En compresión perpendicular el ELP promedio $\left(52.24 \mathrm{~kg} / \mathrm{cm}^{2}\right)$ fue superior al encontrado por Aróstegui y Sato (1970) $\left(46.2 \mathrm{~kg} / \mathrm{cm}^{2}\right)$ e inferior al de JUNAC (1981b) $\left(86 \mathrm{~kg} / \mathrm{cm}^{2}\right)$. Valor que corresponde al $33 \%$ del ELP en com- 
presión paralela (Cuadro 3), aunque JUNAC (1984), especifica que debe variar entre el 20 y el 25\% del mismo.

El MOR promedio en cizalladura radial $\left(49.29 \mathrm{~kg} / \mathrm{cm}^{2}\right)$ fue inferior al reportado por JUNAC (1981b) $\left(116 \mathrm{~kg} / \mathrm{cm}^{2}\right)$ y al obtenido en cizalladura tangencial $\left(56.38 \mathrm{~kg} / \mathrm{cm}^{2}\right)$. Así mismo, los valores medios poblacionales para la dureza en extremos $(337.90 \mathrm{~kg})$ y en lados $(212.03 \mathrm{~kg})$, fueron inferiores a los obtenidos por JUNAC (1981b) (677 y $452 \mathrm{~kg}$ ) y por Aróstegui y Sato (1970) (516 y 298 kg) (Cuadro $3)$.

Aunque en los rodales de los dos sitios estudiados (S1 y S2) se llevaron a cabo actividades silviculturales (podas de formación y aclareos) y una rigurosa preselección de los árboles que consideró tamaño, forma y condiciones sanitarias, la presencia de abundantes nudos y grano entrecruzado en todas las trozas (de base a copa), modifico el número total y dificultó muchísimo la elaboración de las probetas evaluadas, pudiendo ser una fuente importante de variación de los resultados obtenidos.

De manera general, los valores obtenidos para las propiedades físico-mecánicas del pino romerón de 22 años en plantaciones fueron bajos e inferiores a los reportados en la literatura, diferencias que pueden explicarse a partir de los distintos métodos e intensidades de muestreo, procedimientos de ensayo, manejo silvicultural (Marín 1998, Cueva et al. 2013, Becerra-Montalvo y Zevallos-Pollito 2014, Bonilla y Alarcón 2015), edad, dimensiones y características de los individuos muestreados, además de las diversas condiciones de crecimiento y procedencias (bosque natural o plantación) de los materiales utilizados, que no permitieron realizar una más amplia y completa comparación de los resultados en la presente investigación. De igual forma, al comparar la densidad de la madera de pino romerón obtenida en este trabajo, con la de otras coníferas que se siembran y comercializan en Colombia, ésta resulta inferior a la del pino ciprés (Cupressus lusitánica Mill) y a la del pino patula (Pinus patula Schiede \& Schltdl. \& Cham.) (Vásquez y
Ramírez 2005), lo que conlleva a un gran reto de investigación en manejo y genética para mejorar las características de su madera.

\section{Conclusiones}

Los valores obtenidos en la determinación de las propiedades físico-mecánicas en la madera de árboles de pino romerón de 22 años en dos sistemas de plantación con manejo silvicultural (S1 y S2), presentaron diferencias significativas entre sitios solo para algunas de las propiedades estudiadas (flexión, compresión paralela, cizalladura tangencial y clavos), sin una tendencia marcada con respecto a uno de los mismos y fueron inferiores a los reportados en árboles de bosque natural en Perú y Ecuador.

Estos valores señalan la poca durabilidad natural de la madera de árboles de pino romerón de 22 años en climas tropicales y su utilidad para construcción liviana no estructural, carpintería de obra (puertas, ventanas, zócalos, tablilla de techo, etc.), mueblería, revestimiento de interiores, elaboración de cajas, formaletería, palillos para dientes y fósforos, depresores linguales, triplex, tableros, pulpa y papel, utensilios de cocina, molduras y juegos didácticos.

\section{Agradecimientos}

A la Institución de Educación Superior Colegio Integrado Nacional Oriente de Caldas IES CINOC, por la donación del material vegetal, financiación e interés en el estudio de la especie. Al Laboratorio de Productos Forestales Héctor Anaya López de la Universidad Nacional de Colombia Sede Medellín, y a su personal por su apoyo y colaboración.

\section{Bibliografía}

Acevedo, MM; Kikata, Y. 1994. Atlas de maderas del Perú. Lima, Perú, Universidad Nacional Agraria La Molina Perú - Universidad de Nagoya Japón. 202 p.

Aranguren, A; Márquez, NJ. 2011. Etnoecología de las especies vegetales de los bosques estacio- 
nalmente secos del Estado Mérida. Ethnobotany Research \& Applications 9:307-323.

Arévalo, R; Londoño, A. 2005. Manual para la identificación de maderas que se comercializan en el departamento del Tolima. CORTOLIMA. Ibagué, Colombia, Universidad del Tolima. 146 p.

Aróstegui, VA; Sato, A. 1970. Propiedades físico-mecánicas y usos de dos especies de Podocarpus ( $P$. rospigliosii Pilger; P. montanus var. meridensis Buchholz y Gray). Revista Forestal del Perú 4(1-2):3-11.

Aróstegui, VA. 1982. Recopilación y análisis de estudios tecnológicos de maderas peruanas. Proyecto PNUD/FAO/PER/81/002 Fortalecimiento de los Programas de Desarrollo Forestal en Selva Central. Lima, Perú. 57 p.

Becerra-Montalvo, V; Zevallos-Pollito, PA. 2015. Determinación del turno de corta de Cedrela odorata L., Retrophyllum rospigliosii Pilger y Prumnopitys harmsiana Pilger a través del estudio dendrocronológico en San Ignacio, región Cajamarca-Perú. El Ceprosimad 02(2): 33-47.

Bonilla, JA; Alarcón, JA. 2015. Turnos técnico y económico de tala para arboles de romerillo blanco en ecuador. Ecología Aplicada 14(2): 127-137. DOI: http://dx.doi.org/10.21704/rea. v14i1-2.89

Cárdenas, LD; Salinas, NR. (eds.). 2007. Libro rojo de plantas de Colombia. Volumen 4. Especies maderables amenazadas: Primera parte. Serie libros rojos de especies amenazadas de Colombia. Bogotá, Colombia, Instituto Amazónico de Investigaciones Científicas SINCHI - Ministerio de Ambiente, Vivienda y Desarrollo Territorial. $232 \mathrm{p}$.

Cueva, N; Vélez, DF; Barrios, A. 2013. Pino romerón (Retrophyllum rospigliosii Pilger. C.N.Page) - especie nativa potencial para la reforestación en zonas altas. Bogotá, Colombia, Corporación Nacional de Investigación y Fomento Forestal CONIF ${ }^{\circledR}$-Ministerio de Agricultura y Desarrollo Rural-MADR. 54 p.

Cueva, N; Trujillo, E. 2016. Biología reproductiva del pino romerón-Retrophyllum rospigliosii
(Pilg.) C.N.Page. Pensilvania, Colombia, Institución de Educación Superior Colegio Integrado Nacional Oriente de Caldas (IES CINOC). $43 \mathrm{p}$.

de Lima, LE; Moreira, JR; Napoli, A; Lima, JT; Trugilho PF; Reis, DF. 2016. Study of the physical properties of Corymbia citriodora wood for the prediction of specific cutting force. Scientia Forestalis 44(111): 701-708. DOI: http://dx. doi.org/10.18671/scifor.v44n111.16

Diez, GM. 2004. Exploración de relaciones simbióticas en raíces de pino romerón (Retrophyllum rospigliosii) (Pilg.) C.N. Page (podocarpaceae) en un fragmento de bosque Andino. Medellín, Colombia, Corporación Autónoma Regional del Centro de Antioquia (CORANTIOQUIA). $51 \mathrm{p}$.

Diez, GM; Osorio, VN; Moreno, HF. 2008. Evaluación de la dependencia micorrizal del pino Romerón (Nageia rospigliosii Pilger) bajo condiciones lumínicas contrastantes. Revista Facultad Nacional de Agronomía 61(2):45544563.

Echenique, MR; Barajas, ML; Pinzón, P; Pérez, M. 1975. Estudio botánico ecológico de la región del río Uxpanapa, Veracruz. No. 1: Características tecnológicas de diez especies. México, INIREB. 66p.

Fondo de Promoción de Exportaciones (PROEXPO). 1970. Maderas Colombianas. Bogotá, Colombia. $117 \mathrm{p}$.

García, HA; Chumbimune, SY; Acevedo, MP; Chavesta, M; Cuellar, JE; Salazar, EJ. 2017. Aptitud de uso del ulcumano (Retrophyllum rospigliosii (Pilg.) C.N. Page), procedente de una plantación de 32 años, mediante la caracterización tecnológica y anatómica. Revista Forestal del Perú 32(2): 78-88. DOI: http://dx. doi.org/10.21704/rfp.v32i2.1039

Gómez, ML. 2010. Fenología reproductiva de especies forestales nativas presentes en la jurisdicción de CORANTIOQUIA, un paso hacia su conservación: Volumen I. Medellín, Colombia, Corporación Autónoma Regional del Centro de Antioquia (CORANTIOQUIA). 228 p. 
Herbert, J; Hollingsworth, PM; Gardner, MF; Mill, RR; Thomas, PI; Jaffré, T. 2002. Conservation genetics and phylogenetics of New Caledonian Retrophyllum (Podocarpaceae) species. New Zealand Journal of Botany (40): 175-188. DOI: https://doi.org/10.1080/002882 $\underline{\text { 5X.2002.9512781 }}$

Icontec (Instituto Colombiano de Normas Técnicas y Certificación). 1969. Maderas. Requisitos de las probetas pequeñas para ensayos físicos y mecánicos de la madera. NTC 301. Bogotá, Colombia. 2 p.

Icontec (Instituto Colombiano de Normas Técnicas y Certificación). 1974a. Maderas. Toma de muestras para ensayos físicos y mecánicos. NTC 787. Bogotá, Colombia. 9 p.

Icontec (Instituto Colombiano de Normas Técnicas y Certificación). 1974b. Maderas. Determinación del peso específico aparente. NTC 290. Bogotá, Colombia. 5 p.

Icontec (Instituto Colombiano de Normas Técnicas y Certificación). 2003a. Maderas. Método para determinar la contracción. NTC 701. Bogotá, Colombia. 4 p.

Icontec (Instituto Colombiano de Normas Técnicas y Certificación). 2003b. Maderas. Determinación de la resistencia a la flexión. NTC 663. Bogotá, Colombia. 5 p.

Icontec (Instituto Colombiano de Normas Técnicas y Certificación). 2004a. Maderas. Ensayos con probetas pequeñas. NTC 3377 . Bogotá, Colombia. 76 p.

Icontec (Instituto Colombiano de Normas Técnicas y Certificación). 2004b. Maderas. Determinación de la resistencia a la compresión axial o paralela al grano. NTC 784. Bogotá, Colombia. 5 p.

Icontec (Instituto Colombiano de Normas Técnicas y Certificación). 2004c. Maderas. Determinación de la resistencia a la compresión perpendicular al grano. NTC 785. Bogotá, Colombia. 5 p.

Icontec (Instituto Colombiano de Normas Técnicas y Certificación). 2004d. Maderas. Determinación de la resistencia al cizallamiento paralelo al grano. NTC 775. Bogotá, Colombia. $3 \mathrm{p}$.

Icontec (Instituto Colombiano de Normas Técnicas y Certificación). 2004e. Maderas. Determinación de la dureza (método Janka). NTC 918. Bogotá, Colombia. 4 p.

Icontec (Instituto Colombiano de Normas Técnicas y Certificación). 2004f. Maderas. Método de extracción de clavos. NTC 951. Bogotá, Colombia. 5 p.

Icontec (Instituto Colombiano de Normas Técnicas y Certificación). 2006. Maderas. Determinación del contenido de humedad para ensayos físicos y mecánicos. NTC 206-1. Bogotá, Colombia. 3 p.

Junta del Acuerdo de Cartagena (JUNAC). 1981a. Descripción general y anatómica de 105 maderas del grupo andino. Cali, Colombia, Talleres Gráficos Carvajal S.A. 441 p.

Junta del Acuerdo de Cartagena (JUNAC). 1981b. Tablas de propiedades físicas y mecánicas de la madera de 24 especies de Colombia. Lima, Perú, JUNAC PADT- REFORT. 54 p.

Junta del Acuerdo de Cartagena (JUNAC). 1983. Secado y preservación de 105 maderas del Grupo Andino. Lima, Perú, JUNAC PADTREFORT. $152 \mathrm{p}$.

Junta del Acuerdo de Cartagena (JUNAC). 1984. Manual de diseño para maderas del Grupo Andino. Lima, Perú, JUNAC PADT- REFORT. 586 p.

Junta del Acuerdo de Cartagena (JUNAC). 1989. Manual del grupo Andino para el secado de maderas. Lima, Perú, JUNAC PRID-MADERA. 450 p.

Kollman, F. 1959. Tecnología de la Madera y sus aplicaciones. Madrid, España, Ministerio de Agricultura, Dirección General de montes, caza y pesca fluvial, Instituto Forestal de Investigaciones, Experiencias y Servicios de la madera. $675 \mathrm{p}$.

Lima, IL; Longui, EL; Freitas, MLM; Zanatto, ACS; Zanata, M; Florsheim, SMB; Bortoletto, G. 2014. Physical-mechanical and anato- 
mical characterization in 26-year-old Eucalyptus resinifera wood. Floresta e Ambiente, Seropédica 21(1): 91-98. DOI: http://dx.doi. org/10.4322/floram.2014.006

Marín, A. 1998. Ecología y silvicultura de las Podocarpáceas andinas de Colombia. Cali, Colombia, Departamento de Investigación Forestal, Smurfit Cartón de Colombia S.A. 143 p.

Ordóñez, VR; Bárcenas, GM; Quiroz, A. 1990. Características físico-mecánicas de la madera de diez especies de San Pablo Macuiltianguis, Oaxaca. Boletín Técnico La Madera y su Uso no. 21. México, Instituto de Ecología - UAM. 30 p.

Pedroso, BH; Rocha, MA; Mendonça, HE; Vinha, AJ; de Sousa, DM; Carneiro, M. 2016. Propriedades físicas da madeira de Calycophyllum spruceanum Benth. em função do diâmetro e da posição (base e topo) no fuste. Scientia Forestalis 44(111): 759-768. DOI: http://dx.doi. org/10.18671/scifor.v44n111.22

Programa Regional para el Desarrollo del Sur (PREDESUR). 1979. Estudio tecnológico de propiedades y usos de 64 especies maderables del sur ecuatoriano. Quito, Ecuador, Publicación $\mathrm{N}^{\circ} 94.117 \mathrm{p}$.

Silba, J. 1983. A new specie of Decussocarpus de Laub. (Podocarpaceae) from Brazil. Phytologia (54): 460-462.

StatPoint Technologies. 2009. STATGRAPHICS $^{\circledR}$ Centurion XVI software version 16.103. Warrenton, Virginia: StatPoint Technologies, Inc. Disponible en www.statgraphics.net

Torres, JH. 1988. Monografía N 5: Podocarpaceae. Flora de Colombia. Bogotá, Colombia, Imprenta Nacional. 75 p.

Vaca, MS. 2003. Impacto de la tala selectiva en los bosques de Podocarpus de San Ignacio, Cajamarca. Lyonia 5(2):143-156.

Vásquez, AM; Ramírez, AM. 2005. Maderas que se comercializan en el Valle de Aburrá. Medellín, Colombia, Área Metropolitana del Valle de Aburrá. 246 p.

Vásquez, AM; Alcántara, VE. 2009. Anatomía y densidad de la madera de árboles de pino romerón [Retrophyllum rospigliosii (Pilger) C.N. Page] de ocho años de edad. Revista Facultad Nacional de Agronomía 62(1):48694880 .

Vásquez, AM. 2010. Anatomía de la madera de las podocarpáceas colombianas. Tesis Ph.D. Córdoba, España, Universidad de Córdoba. $128 \mathrm{p}$.

Vásquez, AM; Alcántara, VE; Herrera, MA. 2010. Wood anatomy of Colombian Podocarpaceae (Podocarpus, Prumnopitys and Retrophyllum). Botanical Journal of the Linnean Society (164): 293-302. DOI: https://doi. org/10.1111/j.1095-8339.2010.01087.x

Veillón, JP. 1962. Coníferas autóctonas de Venezuela: los Podocarpus. Con especial énfasis sobre las podocarpáceas de la región central del estado de Mérida-Venezuela. Mérida, Venezuela, Universidad de los Andes. 159 p.

Vieira, P; Alves, M; Dias, AF; Monteiro, A; do Nascimento, AM. 2016. Qualidade tecnológica da madeira de Corymbia citriodora (Hook.) K.D. Hill \& L.A.S. Johnson submetida ao processo de termorretificação. Scientia Forestalis 44(110): 343-350. DOI: https://dx.doi. org/10.18671/scifor.v44n110.07

Vicuña-Miñano, EE. 2005. Las Podocarpáceas de los bosques montanos del noroccidente peruano. Revista Peruana de Biología 12(2): 283-288. DOI: http://dx.doi.org/10.15381/rpb. v12i2.2400

Zenteno-Ruíz, FS. 2007. Retrophyllum rospigliosii (Podocarpaceae), un nuevo registro de pino de monte, en el noroeste de Bolivia. Kempffiana 3(2): 3-5.

Zevallos, P. 1988. Estudio dendrológico de las Podocarpáceas y otras especies forestales de Jaén y San Ignacio. Lima, Perú, Ed. Gráfica Bellido. $71 \mathrm{p}$. 